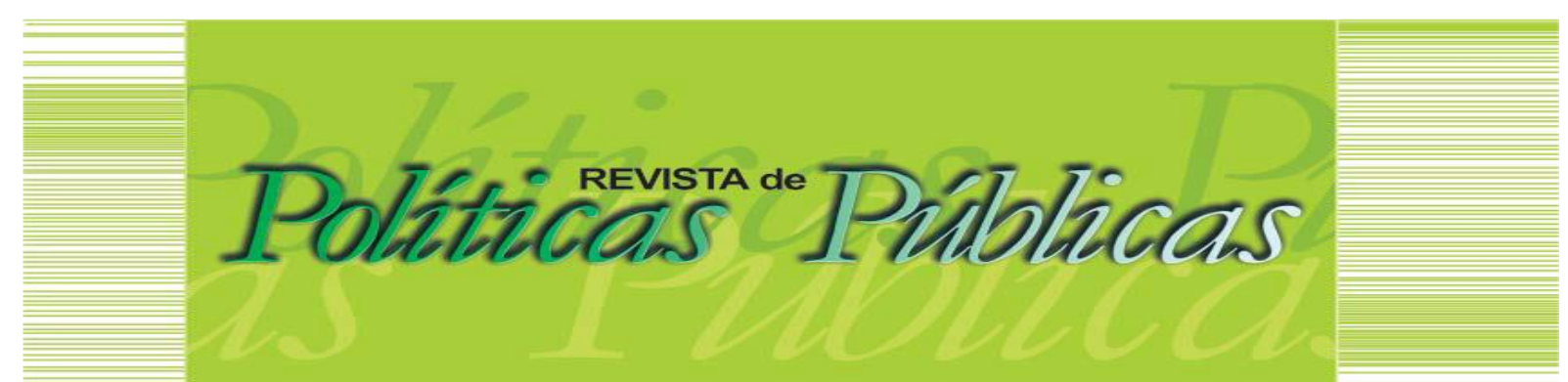

\title{
PANDEMIA DE COVID-19 E PROMOÇÃO DA SAÚDE DO IDOSO NA PERSPECTIVA DE TRABALHADORES DA SAÚDE
}

\author{
Xavéle Braatz Petermann ${ }^{1}$ \\ Sheila Kocourek ${ }^{2}$
}

\section{Resumo}

Este estudo teve como objetivo analisar a influência da pandemia de COVID-19 na promoção da saúde do idoso na perspectiva de trabalhadores da saúde da atenção primária. Trata de um estudo de caso, transversal e qualitativo desenvolvido em um município de pequeno porte do interior do Estado do Rio Grande do Sul. Participaram sete trabalhadores, sendo a amostra intencional, por conveniência e saturação. Os dados foram coletados mediante grupo focal e entrevistais individuais e analisados por meio da análise temática. As categorias que denotaram a influência da pandemia de COVID-19 na promoção da saúde do idoso compreenderam o distanciamento da rede de suporte social e a rede de apoio familiar. Os resultados encontrados retrataram o impacto da pandemia na vida dos idosos, em especial na saúde mental e emocional, sendo necessárias diretrizes de continuidade das ações de promoção da saúde.

Palavras-chave: Promoção da Saúde. Idoso. COVID-19. Trabalhador de saúde. Atenção Primária à Saúde.

\section{PANDEMIC OF COVID-19 AND HEALTH PROMOTION OF THE ELDERLY FROM THE HEALTH WORKERS PERSPECTIVE}

\begin{abstract}
This study aimed to analyze the influence of the COVID-19 pandemic on the promotion of elderly health from the perspective of primary health care workers. This is a case study, cross-sectional and qualitative developed in a small city in the interior of the state of Rio Grande do Sul. Seven workers participated, the sample being intentional, for convenience and saturation. Data were collected through a focus group and individual interviews and analyzed through thematic analysis. The categories that denoted the influence of the COVID-19 pandemic in promoting the health of the elderly included the distance from the social support network and the family support network. The results found portrayed the impact of the pandemic on the lives of the elderly, especially on mental and emotional health, requiring guidelines for the continuity of health promotion actions.

Keywords: Health Promotion. Elderly. COVID-19. Health worker. Primary Health Care.
\end{abstract}

Artigo recebido em: 10/08/2020 Aprovado em: 27/05/2021 DOI: http://dx.doi.org/10.18764/2178-2865.v25n1p199-211

\footnotetext{
1 Fisioterapeuta. Mestre em Gestão de Organizações Públicas pela Universidade Federal de Santa Maria (UFSM). Fisioterapeuta do Quadro de Funcionários da Secretaria de Saúde da Prefeitura Municipal de Arroio do Tigre (PMAT). Email: xavelepetermann@gmail.com

2 Assistente Social. Doutora em Serviço Social pela Pontifícia Universidade Católica do Rio Grande do Sul (PUCRS). Professora do Quadro de Colaboradores do Programa de Pós-Graduação em Gestão de Organizações Públicas (PPGOP) da Universidade Federal de Santa Maria (UFSM). E-mail: sheilakocourek@gmail.com
} 


\section{INTRODUÇÃO}

O primeiro caso de COVID-19 foi identificado em Wuhan na China em dezembro de 2019, e, depois disso, ocorreu um progressivo aumento do número de casos e óbitos em diversos países. Diante desse cenário, a Organização Mundial da Saúde (OMS) declarou que a COVID-19 se caracterizava como uma Emergência de Saúde Pública de Importância Internacional em 30 de janeiro de 2020 (OMS, 2020). No Brasil, a epidemia foi assumida como uma Emergência em Saúde Pública de Importância Nacional em três de fevereiro de 2020 (BRASIL, 2020a).

Em meio à atual pandemia as pessoas idosas são apontadas como o grupo populacional que requer maior atenção e cuidado, principalmente aqueles com condições crônicas (HAMMERSCHMIDT; SANTANA, 2020), uma vez que o aumento da idade está associado à maior mortalidade (ZHOU et al., 2020). Devido a isso, foi recomendado como medida de proteção a adoção do distanciamento social, sobretudo deste grupo etário (BRASIL, 2020b).

Entretanto, Hammerschmidt e Santana (2020) salientam que é preciso a compreensão de que 0 distanciamento social do idoso não caracteriza 0 seu abandono, sendo que cada família em conjunto com o idoso deve discutir estratégias de proteção e enfrentamento. Ainda, esses autores (HAMMERSCHMIDT; SANTANA, 2020), destacaram que mesmo aqueles idosos que residem sozinhos necessitam de apoio de pessoas de referência para abarcar suas necessidades e demandas do cotidiano.

Ornell et al. (2020) apontam como estratégias para a proteção do idoso 0 auxílio nas tarefas que devem ser realizadas em locais de risco, manter maior vigilância em relação aos sintomas e fornecer suporte emocional. Para esse suporte, o Ministério da Saúde (BRASIL, 2020c) recomendou aos gestores públicos que durante a atual pandemia seja fortalecida a rede de apoio familiar da pessoa idosa como estratégia prioritária de cuidado à saúde do idoso.

Quando se refere à saúde da população idosa, duas políticas públicas devem ser consideradas como norteadoras das ações voltadas para esse grupo etário nos serviços de atenção primária, a Política Nacional de Promoção da Saúde - PNPS (BRASIL, 2014) e a Política Nacional de Saúde da Pessoa Idosa - PNSPI (BRASIL, 2006). A PNPS destaca especial relevância às ações voltadas os idosos e a PNSPI aponta para a organização do sistema de saúde, com a finalidade de promover, manter e recuperar a capacidade funcional dos idosos, com destaque para as atividades de promoção da saúde do idoso.

A preocupação com esta temática - promoção da saúde do idoso e COVID-19 - emergiu de questionamentos frente aos diversos arranjos familiares em que a pessoa idosa está inserida e a carga de estresse emocional devido à insegurança e incerteza em meio à pandemia, em que a maior 
vulnerabilidade é atribuída aos idosos. Sendo que, todos esses aspectos possuem interface com questões sociais, culturais e econômicas do contexto de vida do idoso.

Ainda, muitos idosos vivem em condições extremas de pobreza, promovem o sustento da família, estão acamados, possuem mobilidade reduzida, com síndromes demências, vivem sozinhos, residem em instituições de longa permanência ou estão em situação de rua, entre outras tantas realidades. Diante desse cenário, é preciso refletir sobre o cuidado dos idosos, com estratégias para a continuidade e fortalecimento das ações ligadas à promoção da saúde.

Sendo assim, este estudo teve como objetivo analisar a influência da pandemia de COVID-19 na promoção da saúde da pessoa idosa na perspectiva de trabalhadores da saúde de equipes de atenção primária.

\section{METODOLOGIA}

Este estudo apresenta um recorte de uma pesquisa de mestrado profissional sobre as ações da política de promoção da saúde direcionadas para a população idosa na perspectiva de trabalhadores da saúde das equipes de atenção primária. A abordagem utilizada foi a do tipo qualitativa, transversal, por meio de um estudo de caso, desenvolvido no período de fevereiro a junho de 2020 , em um município de pequeno porte do interior do Estado do Rio Grande do Sul com 12,9\% da população total de idosos em 2010 (IBGE, 2010).

A Rede de Atenção à Saúde (RAS) desse município conta com cobertura populacional de $100 \%$ das equipes de atenção primária, formada pelas equipes de atenção básica, pelas equipes de saúde da família e pelo Núcleo Ampliado de Saúde da Família e Atenção Básica. Esse município conta com Serviço de Atendimento Móvel de Urgência (SAMU) e com serviços especializados de laboratórios contratualizados, convênios com instituições prestadoras de serviços de saúde na área hospitalar, além de serviços de referência de média e alta complexidade estabelecidos pelo Estado.

A amostra foi intencional, por conveniência e saturação, sendo composta por sete profissionais de saúde que atuam na atenção primária, os quais aceitaram participar da pesquisa de maneira voluntária e assinaram o Termo de Consentimento Livre e Esclarecido. Assim, a amostra do estudo foi composta por um nutricionista, um assistente social, um cirurgião-dentista, dois agentes comunitários de saúde e dois enfermeiros (Quadro 1). Para preservar a identidade dos profissionais envolvidos no estudo, optou-se pelo uso de pseudônimos. 
Quadro 1- Caracterização dos profissionais

\begin{tabular}{|clcc|}
\hline Nome & Gênero & $\begin{array}{c}\text { Tempo de serviço no } \\
\text { município }\end{array}$ & $\begin{array}{c}\text { Experiência profissional } \\
\text { anterior }\end{array}$ \\
\hline Safira & Feminino & De três meses a cinco anos & Consultório particular \\
\hline Jade & Feminino & De três meses a cinco anos & Não possui \\
Cristal & Feminino & De 25 a 30 anos & Consultório particular \\
\hline Pérola & Feminino & De cinco a 10 anos & Saúde Pública \\
Citrino & Masculino & De três meses a cinco anos & Não possui \\
\hline Turmalina & Feminino & De três meses a cinco anos & Saúde Pública \\
\hline Ametista & Feminino & De três meses a cinco anos & Saúde Pública \\
\hline
\end{tabular}

Fonte: Dados da pesquisa (2020).

Para participar do estudo os profissionais poderiam ser de ambos os sexos, de todas as idades e estarem envolvidos com ações da política de promoção da saúde para a população na atenção primária, por no mínimo, três meses, para que os sujeitos possam narrar sua percepção a respeito das ações realizadas (AUGUSTO et al., 2001). Foi excluído um sujeito por desenvolver ações com tempo inferior a três meses, o que não permitiria o levantamento de informações pertinentes ao estudo. A amostragem por saturação é usada para definir o tamanho final da amostra de uma pesquisa, que será quando as narrativas dos novos sujeitos da pesquisa pouco acrescentariam aos dados já coletados, cessando a captação de novas narrativas (FONTANELLA et al., 2008).

Os dados foram coletados por meio de grupo focal e entrevistas individuais. A proposta inicial foi a realização de grupos focais com os profissionais, entretanto, frente ao contexto de pandemia, optou-se por entrevistas individuais como medida de proteção. Então, foi realizado um grupo focal com quatro profissionais e três entrevistas individuais com três profissionais. 0 instrumento de coleta de dados foi norteado por um roteiro de questões elaboradas pelas autoras com base na PNPS (BRASIL, 2014) e PNSPI (BRASIL, 2006). Devido à pandemia foi inserido no roteiro mais uma questão norteadora sobre a influência da COVID-19 na promoção da saúde do idoso. Esse roteiro pode ser visualizado no Quadro 2.

Quadro 2 - Roteiro de questões norteadoras.

Caracterização

Aspectos de vida;

Formação e experiência profissional; 


\begin{tabular}{|c|c|}
\hline $\begin{array}{l}\text { Modelo baseado na } \\
\text { PNPS e PNSPI }\end{array}$ & $\begin{array}{c}\text { Conceito de promoção da saúde; } \\
\text { Gestão da política de promoção da saúde; } \\
\text { Promoção da saúde voltada para a população idosa; }\end{array}$ \\
& $\begin{array}{r}\text { Efeito das ações de promoção da saúde direcionada para os idosos; } \\
\text { Planejamento e avaliação; } \\
\text { Facilitadores e dificuldades; }\end{array}$ \\
\hline COVID-19 & Pandemia de COVID-19 e promoção da saúde do idoso; \\
\hline Complementações & Sugestões e críticas. \\
\hline
\end{tabular}

Fonte: elaborado com base na PNPS (BRASIL, 2014) e PNSPI (BRASIL, 2006).

O grupo focal se caracterizou por uma discussão em grupo com os sujeitos da pesquisa e possui como essência a interação entre os sujeitos e o pesquisador (MINAYO, 2010). E, a entrevista consiste em uma conversa com finalidade, orientada por um roteiro e não condicionada a um padrão de alternativas (MINAYO, 2010). O recrutamento dos sujeitos ocorreu por meio de indicações sucessivas de pessoas pertencentes à população de estudo (PELICIONI, 2001).

As ações e programas da política de promoção da saúde que os sete profissionais da atenção primária relataram que desenvolviam para os idosos foram as atividades grupais (hiperdia, fisioterapia, oficina terapêutica, ginástica e grupo de idosos vinculados ao Centro de Referência de Assistência Social - CRAS), a implantação da caderneta do idoso, as orientações nos atendimentos individuais/ visitas domiciliares e os dias de campanha (outubro rosa, novembro azul e setembro amarelo). Considerando os protocolos de distanciamento social ${ }^{1}$, os idosos deixaram de participar dos grupos ofertados pelas equipes da atenção primária e CRAS, bem como se afastaram de amigos, conhecidos e familiares.

A análise dos dados foi por meio da análise temática (MINAYO, 2010). Essa análise buscou descobrir os núcleos de sentido que compõem uma comunicação, cuja presença ou frequência possuem significado para o objeto de estudo. A partir disso, são propostas inferências e realizadas interpretações, inter-relacionando-as com o quadro teórico inicialmente desenhado.

O estudo foi aprovado pelo Comitê de Ética da Instituição de Ensino Superior à qual os autores estão vinculados (CAAE 25985719.9.0000.5346) e está de acordo com a Resolução n 466/13 do Conselho Nacional de Saúde. 


\section{RESULTADOS E DISCUSSÃO}

A categorização que denotou a percepção dos trabalhadores de equipes de atenção primária sobre a influência da COVID-19 na promoção da saúde da pessoa idosa compreendeu duas categorias: "distanciamento da rede de apoio social" e "rede de apoio familiar". Sendo assim, a Figura 1 ilustrou esta perspectiva.

Figura 1 - Percepção dos trabalhadores de saúde sobre a influência da COVID-19 na promoção da saúde direcionada para os idosos

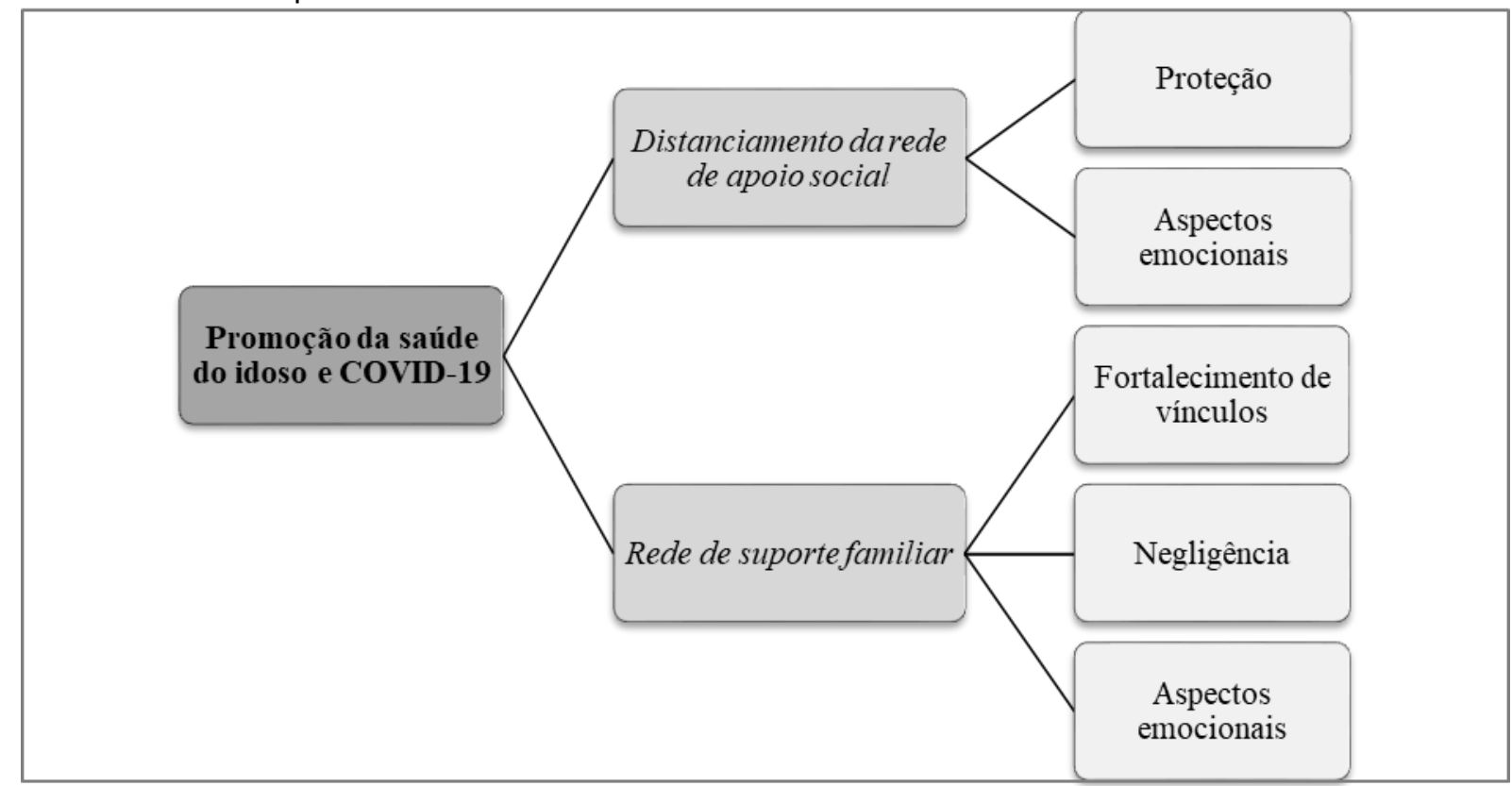

Fonte: Dados da pesquisa (2020).

A Figura 1 retratou que as categorias, bem como os elementos que as elegeram, apresentaram-se inter-relacionadas no contexto da promoção da saúde dos idosos em tempos de COVID-19 e devem ser consideradas em conjunto para o planejamento da continuidade das ações dessa política pública.

\subsection{A rede de suporte social e a promoção da saúde do idoso}

As redes sociais e comunitárias são consideradas como um determinante da saúde do idoso, caracterizando-as como capital social formado por relações de confiança, cooperação e reciprocidade (GEIB, 2012). Em função da pandemia de COVID-19, para a proteção da saúde da pessoa idosa foi recomendado fortemente o distanciamento social. Sendo assim, os idosos deixaram de participar dos grupos ofertados pelas equipes da atenção primária, além disso, também ocorreu um afastamento de amigos, vizinhos e conhecidos. 
As falas dos profissionais retrataram a importância do isolamento social para a proteção do idoso, entretanto, o isolamento pode impactar de maneira negativa na saúde psicológica do idoso:

O idoso está tendo que se isolar para se proteger para não ser infectado pela COVID-19 (...) então considerando esse isolamento social, esse idoso ficou muito mais afastado de quaisquer atividades (...) (Safira).

(...) para a doença realmente quem tiver mais isolado, nós vamos evitar muita coisa, claro porque é proteção. Mas por outro lado, o isolamento acaba prejudicando bastante, eu acredito essa parte do emocional, (...) porque faz falta para eles, ter essa conversa entre eles, se divertir, dar risada (...) (Citrino)

Destacam-se nas narrativas as atividades de lazer e de confraternização, as quais retratam a importância do contexto das relações sociais na saúde dos idosos. Neri e Vieira (2013) descrevem que o envolvimento dos idosos em atividades de natureza social tem influência direta na sua saúde, trazendo benefícios para a cognição, longevidade e funcionalidade e favorecendo sentimentos de utilidade, senso de pertencimento e intercâmbio de ajuda. Corroborando, Guedes et al. (2017) relataram que a percepção do apoio social como determinante importante da saúde da pessoa idosa, ainda, é um grande desafio, não somente nos serviços de saúde, mas também na comunidade, como um instrumento de transformação do processo de vida do idoso. Ainda, os mesmos autores (GUEDES et al., 2017) salientam que o apoio social é apenas uma da várias facetas que devem ser consideradas na atenção à saúde do idoso e não mais somente à doença. A partir disso, percebe-se a relação direta das atividades de apoio social e a saúde da pessoa idosa, em especial a saúde emocional.

O isolamento contribuiu para o aumento da procura de atendimento nas unidades de saúde devido doenças crônicas descompensadas - hipertensão e diabetes - e agravamento de questões relacionadas à saúde emocional.

Esse isolamento, com o passar do tempo e com a chegada do inverno vai agravar mais ainda a saúde dos idosos, idosos com depressão e tentativas de suicídio, acho que a pandemia vai agravar essa situação, porque já tem muitos idosos com depressão que vai se agravar (...) (Cristal).

(...) muitos idosos vem procurar o atendimento de saúde muitos aquela descompensação de pressão e diabetes, crises de ansiedade e até para dar uma fugida de casa também, já percebemos alguns casos de pessoas que eram muito ativas, e daí ele vem no posto só para ver a pressão ou para pedir se podem verificar a pressão. (Ametista).

Dessa maneira, pode-se inferir a interface da rede de apoio social e a saúde da pessoa idosa quando, pelo depoimento de uma profissional, o idoso vem até a unidade de saúde como uma maneira de receber esse apoio. Para muitos idosos, as redes sociais representam o único recurso disponível para compartilhar as cargas da vida cotidiana (GEIB, 2012), buscando suporte emocional e uma percepção mais positiva das condições de vida (GONÇALVES, 2011). Complementando, para 
Guedes et al. (2017), ir à farmácia ou ao mercado vai muito além; nessas atividades o idoso se envolve efetiva e afetivamente em um ambiente social positivo e acolhedor.

A partir das falas dos profissionais, o aumento e agravamento de questões relacionadas à saúde mental e emocional do idoso - ansiedade, depressão e suicídio - decorrente desse isolamento social merecem destaque. Hammerschmidt e Santana (2020) apontam que o distanciamento social é uma medida com intensão de preservar e proteger a saúde da pessoa idosa, entretanto, esse distanciamento social do idoso não caracteriza o seu abandono.

Estudo já apontou o impacto isolamento social na saúde mental dos idosos (ARMITAGE; NELLUMS, 2020), contribuindo para o aumento do risco de doenças cardiovasculares, autoimunes, cognitivas e de saúde mental, com maior risco de depressão e ansiedade (SANTINI et al., 2020). Complementando, outros estudos recentes (SHIGEMURA et al., 2020; GONÇALVES JÚNIOR et al., 2020; KHAN et al., 2020) descrevem o efeito negativo da pandemia na saúde psicológica, destacando a necessidade urgente de abordar os distúrbios emocionais causados devido à situação atual e ao isolamento social.

Nas falas dos profissionais, também, destacaram-se as doenças crônicas descompensadas, como o diabetes e hipertensão arterial. Segundo Ferreira et al. (2020) esse período com maior reclusão domiciliar e comportamento sedentário decorrente do isolamento social pode ser associado com o surgimento e agravamento de comorbidades agregadas a um maior risco cardiovascular, como obesidade, aumento da pressão arterial, intolerância à glicose, bem como transtornos psicossociais como a ansiedade e a depressão.

Jiménez-Pavón et al. (2020) recomendam o exercício físico como um alternativa para combater os consequências mentais e físicas do isolamento em razão do COVID-19 na saúde da pessoa idosa. Os mesmos autores (JIMÉNEZ-PAVÓN et al., 2020) concluem que a manutenção e adoção de um estilo de vida ativo em casa são de extrema importância para a saúde da população, em especial para os idosos.

Ainda, o acesso a informações falsas e notícias de alta mortalidade devido à COVID-19 podem agravar a saúde do idoso, conforme as falas dos profissionais, causando medos e angústias.

\footnotetext{
Ao mesmo tempo se protege a pessoa idosa de contato com portadores de Covid-19, o isolamento contribui para condição de estresse, causando muita ansiedade ao ter acesso a notícias ou informações falsas agravando o quadro de estresse, depressão e sintomas físicos como dor no corpo, perda do apetite e sono. (Turmalina).

(...) eu acho uma coisa que afeta bastante os idosos é o noticiário e a rede social noticiando cada vez mortes e mais mortes (Jade).
} 
Percebem-se sintomas de ansiedade, estresse, depressão, dores crônicas, distúrbios alimentares e insônia acentuada pelas informações falsas e pelo medo de adoecer. Estudo de revisão sobre situações de quarentena apontou uma grande prevalência de efeitos psicológicos negativos, principalmente medo e insônia, associado ao humor rebaixado e irritabilidade aumentada (BROOKS et al., 2000). Ornell et al. (2020) descrevem que o impacto da pandemia de COVID-19 poderá ser ainda maior, caracterizando-a como "pandemia do medo e estresse".

Salienta-se que, por meio da percepção dos profissionais de saúde, o isolamento social constitui um importante meio de proteção da pessoa idosa, entretanto, possui efeitos negativos na saúde deste grupo etário. Dessa maneira, estratégias de promoção da saúde considerando os aspectos ligados a saúde psicológica devem ser pensadas nos serviços de atenção primária. Ainda, nesse contexto complexo de pandemia de COVID-19 e isolamento social, deve-se considerar a rede de apoio familiar do idoso, próxima categoria deste estudo.

\subsection{A rede de apoio familiar e a promoção da saúde da pessoa idosa}

O grupo familiar, assim como o social e comunitário, é considerado um espaço de proteção do idoso, permitindo uma melhoria na qualidade de vida (LEITE et al., 2008). Assim, Hammerschmidt, Bonatelli e Carvalho (2020) apontam para o cuidado da pessoa idosa com desenvolvimento de vínculo e interação de familiares e rede apoio. Neste contexto de pandemia e distanciamento social, o contexto familiar acolhedor contribui com a saúde da pessoa idosa; segundo o relato, o convívio em família favoreceu o fortalecimento dos vínculos afetivos.

\footnotetext{
Eu acho que sempre a família acaba influenciando, (...) ele vai sentir que tem alguém preocupado com ele, é importante ter 0 apoio da família, que as orientações são de ficar mais em casa, inclusive para pegar dinheiro ou remédio na farmácia ou no mercado, se alguém da família pode ir, ele vai se sentir mais agradecido e vai unir mais a família, que às vezes, um trabalha aqui 0 outro ali, eles nem se conversam muito, praticamente vão conversar a noite só, essa questão vai até fortalecer os laços, por que é uma questão de ajudar. (Citrino).
}

Notam-se sentimentos de aproximação, solidariedade e preocupação da família que contribuem de maneira efetiva para a saúde do idoso em meio ao isolamento social. Mota et al. (2010) apontam que esse olhar para o contexto familiar em que o idoso está inserido e não apenas para as questões de doença é importante para uma intervenção efetiva junto à família com idoso. Rabelo; Neri (2015) encontraram relações entre a configuração familiar e condições de saúde dos idosos, o que corrobora os achados da perspectiva dos profissionais de saúde deste estudo. 
Ainda, pela percepção de uma profissional, a rede familiar fortalecida 'faz uma diferença enorme', impactando na funcionalidade do idoso e, como consequência, na sua saúde.

(...) mas ele precisa ter uma rede familiar fortalecida, que isso aqui faz uma diferença enorme, por mais que o idoso more sozinho, ele tem um contexto ao redor que facilita essa autonomia. Quando um idoso ele não tem uma rede social e familiar bem constituída isso afeta a questão de saúde, (...) (Pérola).

O suporte familiar contribui de modo significativo para a manutenção e promoção da saúde do idoso. Devido a isso, no processo de planejamento das ações de promoção da saúde do idoso, é necessária a compreensão do contexto familiar, o que implica o entendimento das questões que envolvem a formação e a dinâmica de funcionamento da família (REIS; TRAD, 2015). Assim, olhar para as relações da rede de apoio familiar do idoso em meio à pandemia é necessário para 0 planejamento das ações da política de promoção da saúde.

Entretanto, os profissionais apontam questões relacionadas à negligência dos familiares com o cuidado do idoso e os grupos de promoção da saúde como suporte a esses idosos. Também, descrevem o idoso como principal provedor dos recursos financeiros da família, indicando essa situação como 'bem difícil'.

(...) aqui no interior temos alguns idosos que não possuem suporte familiar ou possuem pouco suporte da família, mora com o filho, mas não tem praticamente convívio, sabe, e isso tem agravado bastante, como os grupos aqui são muito fortes e é um ambiente de distração, enfim, de promoção da saúde, eles saem, se divertem, eu acho que o impacto, principalmente na saúde mental tem sido bem importante, e vai vir mais coisas ainda, porque vamos demorar a voltar ao normal. (...) Temos muitos casos de negligência da família com 0 cuidado com o idoso, às vezes o filho mora do lado e fica com a aposentadoria e mal alcança comida, então é bem difícil. (Ametista).

Rabelo e Neri (2015), em uma amostra de 134 idosos do Estado da Bahia, encontraram que a maior parte desses idosos eram chefes de família, contribuindo total ou parcialmente para 0 sustento da família. Acredita-se que com a pandemia de COVID-19 essa situação tenha se agravado. A insuficiência familiar contribui para uma maior vulnerabilidade da pessoa idosa e para o declínio na saúde psicológica e funcional (SOUZA et al., 2015).

Somando-se a isso, outra profissional também destaca que a violência contra a pessoa idosa 'me preocupa', conforme a narrativa.

Uma coisa que me preocupa é a violência contra a pessoa idosa, e não falo só em violência física, mas os casos de negligência (...) (Pérola).

Esse cenário de pandemia pode contribuir para um aumento da violência contra a pessoa idosa (MORAES et al. 2020), podendo se manifestar nas formas de violência psicológica, física, sexual, 
patrimonial e institucional, negligência e abuso financeiro (BRASIL, 2020d). Então, nas ações de promoção da saúde do idoso se deve considerar essa abordagem nos serviços de atenção primária.

Salienta-se que todos os aspectos complexos e multifacetários abordados na perspectiva dos trabalhadores das equipes de atenção primária se apresentam inter-relacionados no cotidiano de vida dos idosos e assim devem ser considerados para o planejamento das ações da política de promoção da saúde direcionada para os idosos.

\section{CONSIDERAÇÕES FINAIS}

Com base nos resultados deste estudo, percebeu-se que a influência da pandemia de COVID-19 na promoção da saúde do idoso, na perspectiva de profissionais da saúde das equipes de atenção primária, compreendeu duas categorias, sendo estas: "o distanciamento da rede de suporte social", que denotou o distanciamento como proteção do idoso versus a influência negativa na saúde emocional; e, "a rede de apoio familiar", que representou o fortalecimento de vínculos versus a negligência dos familiares e a interface com a saúde emocional.

As percepções aqui encontradas denotaram a complexidade do tema investigado e 0 impacto da pandemia na vida dos idosos, em especial na saúde mental e emocional. Diante do exposto, são necessárias diretrizes de enfrentamento e continuidade das ações nos contextos dos serviços primários de saúde, reinventando os processos de trabalho com alternativas de promoção da saúde do idoso em meio à pandemia.

Embora este estudo tenha apresentado algumas limitações, permitiu uma visão ampla e profunda sobre o impacto dessa pandemia na vida dos idosos.

\section{REFERÊNCIAS}

ARMITAGE, Richard; NELLUMS, Laura B. COVID-19 and the consequences of isolating the elderly. The Lancet Public Health, v. 5, n. 5, p. e256, 2020.

AUGUSTO, Viviane Gontijo et al. Promoção de saúde em unidades básicas: análise das representações sociais dos usuários sobre a atuação da fisioterapia. Ciência \& Saúde Coletiva, v. 16, p. 957-963, 2011.

BRASIL. Ministério da Saúde, Secretaria de Vigilância em Saúde, Secretaria de Atenção à Saúde, Política Nacional de Promoção da Saúde (PNPS). Revisão da Portaria MS/GM Nº 687, de 30 de março de 2006. 
BRASIL. Ministério da Saúde. Boletim epidemiológico especial COE- COVID19. Semana epidemiológica 21. Disponível em: https://portalarquivos.saude.gov.br/images/pdf/2020/May/21/202005-19---BEE16---Boletim-do-COE-13h.pdf. Acesso em: 24 maio 2020b.

BRASIL. Ministério da Saúde. Sobre a doença. Disponível em: https://coronavirus.saude.gov.br/sobrea-doenca\#como-se-proteger.d. Acesso em: 28 maio 2020c.

BRASIL. Portaria MS/GM n 188, de 3 de fevereiro de 2020. Declara Emergência em Saúde Pública de importância Nacional (ESPIN) em decorrência da Infecção Humana pelo novo Coronavírus (2019nCoV). Diário Oficial da União, 2020a.

BRASIL. Portaria $n^{0} 2.528$, de 19 de outubro de 2006. Aprova a Política Nacional de Saúde da Pessoa Idosa. Diário oficial da União. 2006; 1.

BRASIL. Violência contra a pessoa idosa: vamos falar sobre isso? Perguntas mais frequentes sobre direitos das pessoas idosas. Brasília, DF: Ministério da Mulher, da Família e dos Direitos Humanos; 2020. D

BROOKS, Samantha K. et al. The psychological impact of quarantine and how to reduce it: rapid review of the evidence. The Lancet, 2020.

CENSO IBGE. Disponível em: http://www. censo2010. ibge. gov. br/. Acesso em. 1 maio 2020.

FERREIRA, Maycon Junior et al. Vida Fisicamente Ativa como Medida de Enfrentamento ao COVID19. Arquivos Brasileiros de Cardiologia, n. AHEAD, 2020.

FONTANELLA, Bruno José Barcellos; RICAS, Janete; TURATO, Egberto Ribeiro. Amostragem por saturação em pesquisas qualitativas em saúde: contribuições teóricas. Cadernos de saúde pública, v. 24, p. 17-27, 2008.

GEIB, Lorena Teresinha Consalter. Determinantes sociais da saúde do idoso. Ciência \& Saúde Coletiva, v. 17, p. 123-133, 2012.

GONÇALVES JÚNIOR, Jucier et al. A crisis within the crisis: the mental health situation of refugees in the world during the 2019 coronavirus (2019-nCoV) outbreak. Psychiatry research, p. 113000, 2020.

GONÇALVES, Tonantzin Ribeiro et al. Avaliação de apoio social em estudos brasileiros: aspectos conceituais e instrumentos. Ciência \& Saúde Coletiva, v. 16, n. 3, p. 1755-1769, 2011.

GUEDES, Marcelo Barbosa Otoni Gonçalves et al. Apoio social e o cuidado integral à saúde do idoso. Physis: Revista de saúde coletiva, v. 27, p. 1185-1204, 2017.

HAMMERSCHMIDT, Karina Silveira Almeida; BONATELLI, Lisiane Capanema Silva; DE CARVALHO, Anderson Abreu. Caminho da esperança nas relações envolvendo os idosos: olhar da complexidade sob pandemia do Covid-19.

HAMMERSCHMIDT, Karina Silveira Almeida; SANTANA, Rosimere Ferreira. Saúde do idoso em tempos de pandemia COVID-19. Cogitare Enfermagem, v. 25, 2020.

KHAN, Suliman et al. Impact of coronavirus outbreak on psychological health. Journal of Global Health, v. 10, n. 1, 2020. 
LEITE, Marinês Tambara et al. Idosos residentes no meio urbano e sua rede de suporte familiar e social. Texto \& Contexto-Enfermagem, v. 17, n. 2, p. 250-257, 2008.

MINAYO, María C Souza. O desafio do conhecimento. Pesquisa qualitativa em saúde. 2004.

MORAES, Cláudia Leite . et al. Violência contra idosos durante a pandemia de covid-19 no brasil: Contribuições para o seu enfrentamento. Ciência \& Saúde Coletiva, 2020.

MOTA, Fernanda Rochelly do Nascimento et al. Família e redes sociais de apoio para 0 atendimento das demandas de saúde do idoso. Escola Anna Nery, v. 14, n. 4, p. 833-838, 2010.

NERI, Anita Liberalesso; VIEIRA, Ligiane Antonieta Martins. Envolvimento social e suporte social percebido na velhice. Revista Brasileira de Geriatria e Gerontologia, v. 16, n. 3, p. 419-432, 2013.

ORNELL, Felipe et al. "Pandemic fear" and COVID-19: mental health burden and strategies. Brazilian Journal of Psychiatry, v. 42, n. 3, p. 232-235, 2020.

PELICIONI, Maria Cecilia Focesi et al. A utilização do grupo focal como metodologia qualitativa na promoção da saúde. Revista da Escola de Enfermagem da USP, v. 35, n. 2, p. 115-121, 2001.

RABELO, Doris Firmino; NERI, Anita Liberalesso. Tipos de configuração familiar e condições de saúde física e psicológica em idosos. Cadernos de saude publica, v. 31, p. 874-884, 2015.

REIS, Luciana Araújo; TRAD, Leny Alves Bonfim. Suporte familiar ao idoso com comprometimento da funcionalidade: a perspectiva da família. Revista Psicologia-Teoria e Prática, v. 17, n. 3, 2015.

SANTINI, Ziggi Ivan et al. Social disconnectedness, perceived isolation, and symptoms of depression and anxiety among older Americans (NSHAP): a longitudinal mediation analysis. The Lancet Public Health, v. 5, n. 1, p. e62-e70, 2020.

SHIGEMURA, Jun et al. Public responses to the novel 2019 coronavirus (2019-nCoV) in Japan: Mental health consequences and target populations. Psychiatry and clinical neurosciences, v. 74, n. 4, p. 281, 2020.

SOUZA, Alessandra de et al. Concepto de insuficiencia familiar en lo adulto mayor: análisis crítico de la literatura. Revista Brasileira de Enfermagem, v. 68, n. 6, p. 1176-1185, 2015.

WHO EMERGENCY COMMITTEE et al. Statement on the second meeting of the International Health Regulations (2005) Emergency Committee regarding the outbreak of novel coronavirus (COVID-19). Geneva: WHO, 2020.

ZHOU, Fei et al. Clinical course and risk factors for mortality of adult inpatients with COVID-19 in Wuhan, China: a retrospective cohort study. The lancet, 2020.

\section{Nota}

10 modelo de distanciamento social adotado foi o 'Modelo do distanciamento controlado' proposto pelo governo do Estado do Rio Grande do Sul. Esse modelo foi baseado na segmentação regional e setorial, prevendo quatro níveis de restrição, representados por bandeiras nas cores amarela, laranja, vermelha e preta, que irão variar de acordo com a propagação da doença e a capacidade do sistema de saúde em cada uma das regiões pré-determinadas. 